\title{
Effects of subsurface barriers on seawater intrusion and nitrate accumulation in coastal aquifers
}

\author{
Qiguo Sun ${ }^{1}$, Tianyuan Zheng ${ }^{1}$, Xilai Zheng ${ }^{1}$, and Marc Walther ${ }^{2}$ \\ ${ }^{1}$ Ocean University of China \\ ${ }^{2}$ Technische Universität Dresden
}

February 5, 2021

\begin{abstract}
The subsurface barrier is one of various engineering measures used to prevent seawater intrusion in coastal regions which has been widely applied. However, its two common types, the cut-off wall and the subsurface dam, are both found to cause nitrate (NO3-) accumulation in landward aquifers. In this study, numerical simulations were conducted to investigate the mechanism of NO3- accumulation caused by the two types of subsurface barriers, as well as the influence of several key parameters, i.e. the infiltration NO3- concentration, the inflow DOC concentration, the barrier height and the barrier location on the performance of the subsurface barriers. The results showed that the cut-off wall generally requires a large height to ensure a satisfactory seawater prevention effectiveness, and it is more likely to cause NO3- accumulation compared to a subsurface dam. On the other hand, despite the subsurface dam may not result in the significant increase of NO3- concentration in groundwater upstream, it cannot be applied to the areas where SI has occurred due to the residual seawater problem. Moreover, the construction of a cut-off wall results in a stagnation zone appeared at the upper corner of the barrier, where accumulated NO3- significantly. With the increase of the barrier height, the stagnation zone expanded, leading to further increase of mean NO3- concentration in the landward aquifer. Since the construction of a subsurface dam will not generate such a zone, the subsurface dam generally has little impact on NO3- accumulation.
\end{abstract}

\section{Hosted file}

Manuscript.pdf available at https://authorea.com/users/393958/articles/507451-effects-ofsubsurface-barriers-on-seawater-intrusion-and-nitrate-accumulation-in-coastal-aquifers 